\title{
Non-local order in gapless systems: Entanglement Spectrum in Spin Chains
}

\author{
Ronny Thomale ${ }^{1}$, D. P. Arovas ${ }^{2}$, and B. Andrei Bernevig ${ }^{1}$ \\ 1 Department of Physics, Princeton University, Princeton, NJ 08544, USA and \\ 2 Department of Physics, University of California at San Diego, La Jolla, California 92093, USA
}

(Dated: October 28, 2018)

\begin{abstract}
We show that the entanglement spectrum can be used to define non-local order in gapless spin systems. We find a gap that fully separates a series of generic, high 'entanglement energy' levels, from a flat band of levels with specific multiplicities that uniquely define the ground-state, and remains finite in the thermodynamic limit. We pick the appropriate set of quantum numbers, and then partition the system in this space. This partition corresponds to a very non-local real-space cut. Despite the fact that the Laughlin state is bulk gapped while the antiferromagnetic spin chain state is bulk gapless, we show that the $S=\frac{1}{2}$ Heisenberg antiferromagnet in one dimension has an entanglement spectrum almost identical to that of the Laughlin Fractional Quantum Hall state in two dimensions, revealing the similar field theory of their low-energy edge and bulk excitations respectively. In addition, we investigate the dimerization transition from the perspective of entanglement gap scaling.
\end{abstract}

PACS numbers: 73.43.2̌013f, 11.25.Hf

Gapped topological phases of matter usually lack local order parameters that can distinguish them from trivial ones. In the presence of a gap in the excitation spectrum, several non-local indicators of the topological nature of the topological phase, such as ground-state degeneracy on compact high-genus manifolds [1] as well as the structure of edge modes and their scaling exponents [2] exist, but do not fully describe the topological phase. In the absence of an excitation gap, the nature of quantum order is much less understood, especially in systems lacking a local order parameter. For example, torus degeneracy cannot distinguish gapless phases, since the mixing of infinitesimally low-energy excitations can lead to many new states quasi-degenerate with the true ground-state. There are several examples of gapless phases of matter, most notably in one-dimension (but also in higher dimensions [3]), which are known to display highly non-trivial properties. The parade example is the spin- $\frac{1}{2}$ Heisenberg chain, a spin liquid which exhibits new elementary excitations, the spinons [4, 5], which fractionalize out of the usual antiferromagnetic spin wave. While for gapped systems such as the AKLT spin chain, non-local order parameters have been found, no similar quantity exists for gapless spin-disordered chains.

The advent of quantum information theory has led to new concepts, such as quantum entanglement, which have proved useful in further characterizing quantum order. The majority of research to date has concentrated on entanglement entropy of a state $\left|\psi_{0}\right\rangle$. Specifically, let the Hilbert space for an $N$-body system be written as a direct product $\mathcal{H}=\mathcal{H}_{A} \otimes \mathcal{H}_{B}$. It is easiest to imagine real space partitions, where the division corresponds to some prescribed real space 'cut'. Then $\rho_{A} \equiv \operatorname{Tr}_{B}(\rho)$, where $\rho=\left|\psi_{0}\right\rangle\left\langle\psi_{0}\right|$, is the reduced density matrix for the $A$ component. The quantity $S_{A}=-\operatorname{Tr}_{A}\left(\rho_{A} \ln \rho_{A}\right)$ is the entanglement entropy, which provides a measure of the quantum entanglement of $\left|\psi_{0}\right\rangle$ with respect to the partitioning $(A, B)$.

Different scalings of this quantity with the size of $A$ for gapped and gapless systems have been proposed and proved [6]. Still, the entanglement entropy remains a single number, and can only provide a limited characterization of topological order. To this end, Li and Haldane $(\mathrm{LH})$ in a recent insightful paper [7] proposed and numerically substantiated that the entanglement spectrum, i.e. the full set of eigenvalues of $\rho_{A}$, partitioned with respect to the equator of the quantum Hall sphere [8], provides a near complete picture of the topological order in $\nu=\frac{5}{2}$ fractional quantum Hall effect (FQHE) states. Writing the eigenvalues of $\rho_{A}$ as $e^{-\xi_{i}}$, where $\xi_{i}$ is an entanglement level, LH showed that the low level spectrum for generic gapped $\nu=\frac{5}{2}$ states exhibited a universal structure, related to conformal field theory, and separated from a non-universal high energy spectrum by an entanglement gap which was finite in the thermodynamic limit. This gap itself was proposed as a 'fingerprint' of the topological order present. It was subsequently shown that the entanglement spectrum can meaningfully distinguish among states which have similar finite size overlap with $\left|\psi_{0}\right\rangle$ but different edge structures [9]. Other bulkgapped phases such as topological insulators have also been shown to reflect their nontrivial nature in their entanglement spectra [10, 11].

In this Letter, we show how to use the entanglement spectrum to characterize non-local order in gapless systems and phases of matter, with emphasis on spin- $\frac{1}{2}$ spin chains. We find that simple real space partitions fail to reveal the nontrivial fundamental structure in these phases. Rather, we find the cut must be made in Fourier (momentum) space. The local cut in momentum space corresponds to a highly non-local cut in real space. We only use the ground-state wavefunction of the system and do not assume any knowledge regarding the gapless ex- 
citation spectrum. If the system is in the $\mathrm{SU}(2)_{1}$ Wess Zumino Witten (WZW) universality class for spin- $\frac{1}{2}$, we show that the low-entanglement level part of the entanglement spectrum has a special form similar to that of the Laughlin 12] FQHE state. The high entanglement levels are separated by a large entanglement gap from the low entanglement WZW portion of the spectrum. This recapitulates the situation for the ground state of the Coulomb Hamiltonian on the quantum Hall sphere [8], but with two important differences: (i) we are here dealing with a spin system, and (ii) we find a complete entanglement gap at all values of the total momentum, whereas LH found a gap only for a finite range of angular momentum $L^{z}$. We numerically show that the entanglement gap is finite in the thermodynamic limit for the Heisenberg chain. As one result of our work, one can classify whether a generic ground-state spin- $\frac{1}{2}$ wavefunction is in the WZW universality class, without resorting to often unreliable computations of critical exponents. We also show that the entanglement spectrum is sensitive withe respect to the dimerization transition - the flow of entanglement levels matches the field theory prediction for the canceling of a marginal operator at exactly the dimerization transition.

We consider spin- $\frac{1}{2}$ spin chains on even-membered rings with periodic boundary conditions (PBCs). The $N$ sites are placed on a circle of radius unity and are hence described by the $N^{\text {th }}$ roots of unity: $z_{j}=e^{2 \pi i j / N} ; j \in$ $\{1, \ldots, N\}$ (see Fig. 11). Without loss of generality, any ground state wavefunction of an SU(2)-invariant Hamiltonian can be written in the form

$$
\left|\Psi_{0}\right\rangle=\sum_{j_{1}, \ldots, j_{K}} \psi\left(z_{j_{1}}, \ldots, z_{j_{K}}\right) S_{j_{1}}^{-} \cdots S_{j_{K}}^{-}|\mathrm{F}\rangle
$$

where $|F\rangle=|\uparrow \ldots \uparrow\rangle$ is the ferromagnetic state. The sum extends over all ways to distribute the $K=\frac{1}{2} N$ down-spins on the ring, and the weights $\psi\left(z_{j_{1}}, \ldots, z_{j_{K}}\right)$ depend only on the position of the spin $\downarrow$ sites (HolsteinPrimakoff representation). We further presume that $\left|\Psi_{0}\right\rangle$ is a translationally invariant total spin singlet (otherwise, in most cases, the state could already be characterized by some other local order parameter). In particular, these are also the conditions for spin liquid states. Mathematically stated, we require $\sum_{j} S_{j}^{-}\left|\Psi_{0}\right\rangle=0$ and

$$
\psi\left(z_{j_{1}+1}, \ldots, z_{j_{K}+1}\right)=e^{i Q} \psi\left(z_{j_{1}}, \ldots, z_{j_{K}}\right),
$$

where $Q=0$ or $\pi$ is the total crystal momentum, depending on whether $K$ is even or odd, respectively. Note that the last condition is a generic property independent of the particular ground-state wavefunction studied. Spin rotational invariance also implies that the wavefunction is invariant under interchange of $\uparrow$ and $\downarrow$ coordinates.

Typically, calculations of entanglement and entanglement entropy proceed by cutting the system spatially into

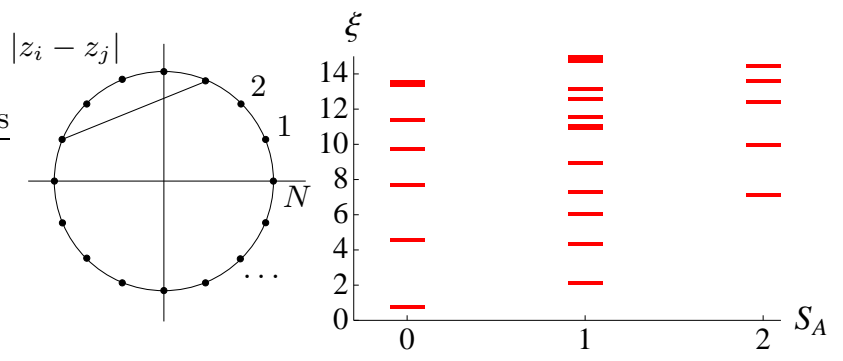

FIG. 1: (color online) Left: Unit circle description of a spin chain with PBCs; the sites lie at the $N^{\text {th }}$ roots of unity. The chord distance specifies the spatial separation of any two sites $z_{i}$ and $z_{j}$. Right: Typical low-level entanglement spectrum for a spatially bisected $N=30$ Heisenberg chain (ground state). Entanglement levels along the vertical axis are plotted versus total spin $S_{A}$ quantum number. Each level has a $2 S_{A}+1$ multiplet degeneracy. No immediate structure is visible.

two disconnected parts and then computing some combination of the eigenvalues of the reduced density matrix which decomposes in independent sectors indexed by the values of the total $S^{z}$. If we choose to perform this cut, compute the eigenvalues of the density matrix, and plot them versus the good multiplet quantum number $S_{A}$, one observes an unremarkable structure such as shown in Fig. 1. To obtain relevant information, one must then analyze the distribution of eigenvalues of the entanglement spectrum [13] or perform calculations of the entanglement entropy [6, 14]. This suggests that a spatial cut is not the most useful way to understand the underlying structure of the spin chain, as no differences in the systems can be suitably resolved [13].

However, real space coordinates are only one option of a set of good quantum numbers in the system. We can also Fourier transform the spin operator on each site and go to a magnon-type description. We define the Fourier transform of the spin flip operators $\tilde{S}_{m}^{-}=\frac{1}{N} \sum_{j} z_{j}^{m} S_{j}^{-}$. The ground state is then written as

$$
\left|\Psi_{0}\right\rangle=\sum_{m_{1}, \ldots, m_{K}} \tilde{\psi}\left(m_{1}, \ldots, m_{K}\right) \tilde{S}_{m_{1}}^{-} \cdots \tilde{S}_{m_{K}}^{-}|\mathrm{F}\rangle,
$$

where

$\tilde{\psi}\left(m_{1}, \ldots, m_{K}\right)=N^{-K} \sum_{j_{1}, \ldots, j_{K}} z_{j_{1}}^{m_{1}} \cdots z_{j_{K}}^{m_{K}} \psi\left(z_{j_{1}}, \ldots, z_{j_{K}}\right)$.

We stress that while the momenta $m_{i}$ also belong to the set $m \in\{1, \ldots, N\}$, the momentum space spin flips do not behave as hard core bosons.

The non-orthogonal momentum state basis can thus be represented by bosonic occupation numbers $n_{m}$ for crystal momentum $m$. The total particle number is $K=\sum_{m} n_{m}$, and $e^{i Q}=\prod_{m} e^{2 \pi i m n_{m} / N} \equiv e^{2 \pi i M / N}$, where $0 \leq n_{m} \leq K \forall m$. For generic wavefunctions, there exist multiple total momentum sectors separated by $2 \pi$, although special spin-chain wavefunctions can have 

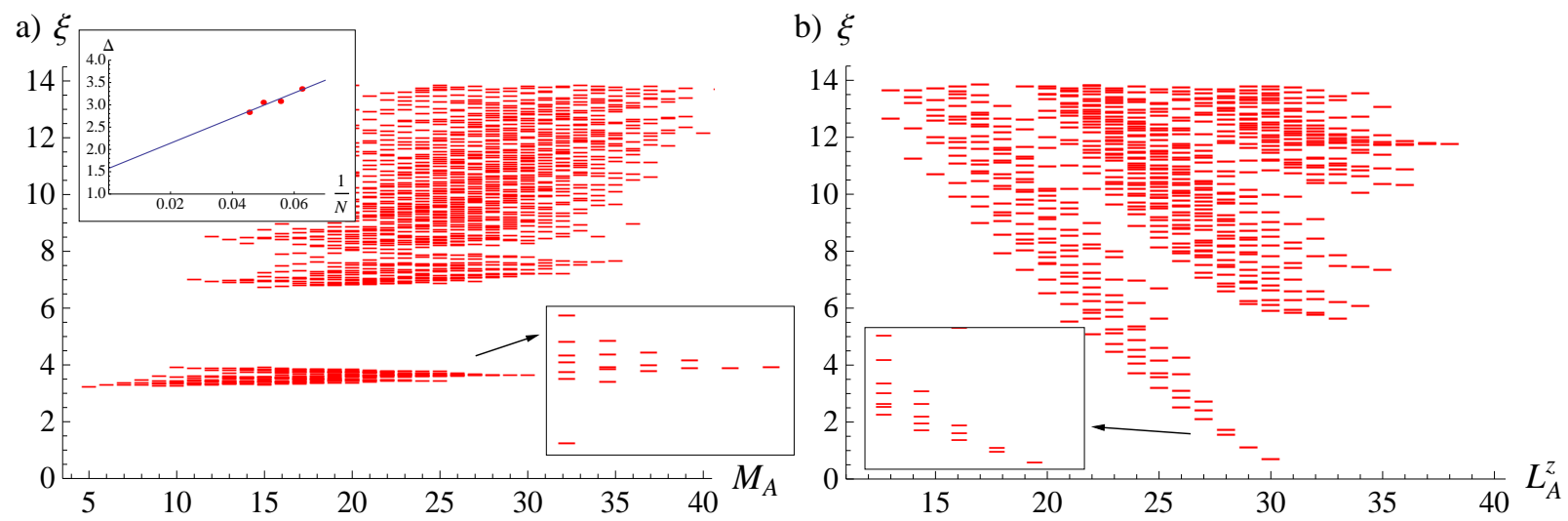

FIG. 2: (color online) a) Entanglement spectrum of the Heisenberg model for $\mathrm{N}=22$ sites, with a cut region containing 5 magnons. The eigenvalues $\xi$ are plotted versus the total momentum subspaces of region $A, M_{A}$. One observes a low level part well separated from higher level contributions. This entanglement gap persists in the thermodynamic limit as shown in the upper inset. For the Haldane Shastry spin chain, exactly the same low-lying levels are found, where the higher levels are shifted to infinity. b) Entanglement spectrum of the $K=11$ particle $\nu=1 / 2 \mathrm{FQH}$ Coulomb state for a half cut on the sphere containing 5 particles. The counting of the low level states is exactly the same. The lower insets in (a) and (b) show the analogous level counting 1,1,2,3,5,7 for the two states.

compact support, i.e. weight in only one of these sectors. We normalize the states by the bosonic normalization of the magnon basis. Now consider a cut in momentum along the middle of the momentum orbitals. Both separate regions $A$ and $B$ can be decomposed with respect to number of particles and total momentum, which is subject to the constraint $N_{A}+N_{B}=K$ and $M_{A}+M_{B}=M$.

Let us first consider the ground state of the $S=\frac{1}{2}$ Heisenberg model, $H=\sum_{j} \vec{S}_{j} \cdot \vec{S}_{j+1}$, for $N=22$ sites. For systems of this size, the explicit Fourier transformation Eq. 4 exceeds numerical limits. Instead, we diagonalize the spin Hamiltonian directly in momentum space, with matrices containing up to several billions of scattering elements. For the Heisenberg ground state, we find that $98 \%$ of the weight of the state is present in the sector with $M=K^{2}$. In the thermodynamic limit, we conjecture that the same low-energy structure of the entanglement spectrum will be present in all momentum sectors. The decomposition of the Heisenberg ground state in momentum notation for a middle cut is presented in Fig. 2, We first observe a low entanglement level part clearly separated from levels higher up. For the low-lying states, from right to left, we observe the counting $1,1,2,3,5,7$ of levels of a $\mathrm{U}(1)$ boson mode until we hit a limit due to finite size of the system. This counting is equivalent to that for the excitations of the low energy field theory for the spin- $\frac{1}{2}$ Heisenberg chain (the SU(2) 1 field theory is equivalent to that of a free $\mathrm{U}(1)$ boson, whose level counting is equal to the number of partitions of its momentum above the ground-state). We then compare to the entanglement spectrum of the Coulomb FQHE state on the sphere with the same number of particles. The $L_{A}^{z}$ quantum number on the quantum Hall sphere corresponds to the total momentum $M_{A}$ for the cut region, where the convention is that the momentum counting starts from the cut, i.e. for maximum $M_{A}$, the occupied momentum orbitals have maximal orbital distance to the cut. This way, equal number of particles beyond the cut and equal absolute values of $M_{A}$ and $L_{A}^{z}$ specify the same configurations in bosonic notation. For the Heisenberg ground state on the chain and the Coulomb state on the sphere, the entanglement spectra are similar both by level sequence as well as by state counting, except that in the spin chain case, a full entanglement gap can be defined between all the $\mathrm{SU}(2)_{1}$ low entanglement levels and the higher lying ones (Fig. 21). A finite size scaling analysis of the entanglement gap for the Heisenberg ground state is shown in the inset of Fig. 2a. Despite the relatively small number of sites that are available to us on small computers, we are confident that the entanglement gap remains finite in the thermodynamic limit.

The entanglement property appears to be intimately linked to the low-energy properties of the system. Other regimes of the spin- $\frac{1}{2}$ system possess different signatures of their entanglement spectrum. Adding a secondneighbor interaction, $H=\sum_{j} \vec{S}_{j} \cdot \vec{S}_{j+1}+\lambda \vec{S}_{j} \cdot \vec{S}_{j+2}$, we find that as we shift from the Heisenberg point $(\lambda=0)$ to the Majumdar-Ghosh [15] point $\left(\lambda=\frac{1}{2}\right)$, where the ground state is a dimer crystal, the entanglement spectrum significantly rearranges, reflecting the failure of the gapless $U(1)$ boson description of the problem in the dimerized phase. From the Sine-Gordon description of the model [16] that we know that the leading logarithmic operator corrections vanish at the dimerization point. This corresponds to the vanishing of the Sine-Gordon cosine term at $\lambda_{c}$. At this critical point, only subleading operators remain, and the theory should look more like that of a free-boson than even the Heisenberg point. We 
find this is manifested in the entanglement spectrum. Upon increasing frustration from the Heisenberg point $\lambda=0$, we find that the lowest (i.e. leading correction) high-energy levels first move upwards, which yields an increase of the entanglement gap $\Delta$ (Fig. 3 $\mathrm{a}$ ). The dimerization transition is given by the vanishing of the leading marginal operator and hence by the maximum entanglement gap. Under this hypothesis, we obtain a value fo $J_{2} / J_{1}=0.215$ in the thermodynamic limit. This is close to the established 0.24 value and even more noteworthy as our largest size system is 22 sites. As the coupling of the Sine-Gordon cosine term changes sign at $\lambda_{c}$, the term is responsible to generate the dimerization gap. This is seen in the entanglement spectrum by the reduction of the entanglement gap, as the dimerization constant $\lambda$ is tuned across its critical value. We remark that our numerical effort was heavy: brute-force Fourrier transform of the wavefunction was possible only up to 18 sites. We then re-formulated our hamiltonian in HolsteinPrimakoff basis, linearized, and introduced a term that gives large energy for double boson occupancy on site. We were then able to reach 22 sites, with non-sparce matrices of several billion matrix elements.

Beyond the same state counting of the universal low level portions of their entanglement spectra, there are several other analogies between the Heisenberg and Laughlin states. As was first discovered for quantum Hall trial states [17], we likewise find for the Heisenberg state that, to a large extent, the weights of the different basis states obey a "product rule" in the sense that the weights of a Heisenberg state of size $N$ can be composed out of weight products of sub-parts of the bosonic momentum state configuration $\left\{m_{1}, \ldots m_{K}\right\}$, given by Heisenberg states of accordingly smaller system size.

This strong structural similarity between the Heisenberg state and Laughlin state is not coincidence. The Heisenberg chain is in the same universality class as the Haldane-Shastry (HS) chain [18, 19], whose ground state wavefunction is $\Psi\left(z_{1}, \ldots, z_{K}\right)=\prod_{i<j}\left(z_{i}-z_{j}\right)^{2} \prod_{i} z_{i}$. Though of no consequence in real space (where the entanglement spectra for HS are unremarkable), we found that the lattice Fourier transformation over all sites (Eq. 4) of the HS state yields exactly the weights of the monomials $m_{\left\{n_{K}, n_{K-1}, \ldots, n_{1}\right\}}$ in the $K$-particle Laughlin state. Hence the entanglement spectrum of the HS ground state, using a momentum cut, is identical in its counting to the entanglement spectrum of the Laughlin state, as shown in Fig. 2 21]. We stress that while the entanglement levels will be different due to the different normalizations for the spin chain and the QH sphere, the counting is identical. The HS state is known to be the conformal gapless phase of all spin models within the the $\mathrm{SU}(2)_{1} \mathrm{WZW}$ class, with special quantum group symmetries. In terms of the fractionalized spinon excitations, it is the model in which the spinons are free in the sense that they interact only through their fractional statistics. Toward
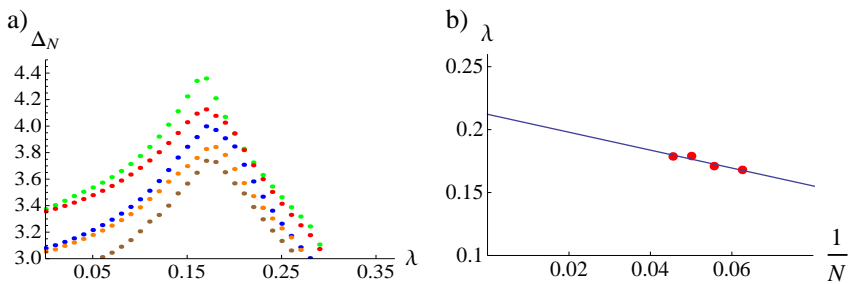

FIG. 3: (color online) a) Entanglement gap $\Delta$ versus dimerization coupling $\lambda$ for different system sizes. As we move away from the Heisenberg point $\lambda=0$, the entanglement gap rise, till it finally shrinks again within the dimerized phase. b) The dimerization transition is estimated from finite size by the $1 / N$ scaling of the $\lambda$ with maximum entanglement gap for different system sizes. The extrapolation gives $\lambda_{c}^{\infty} \sim 0.215$, i.e. in range of $10 \%$ of the exact result from $\mathrm{CFT}$.

the Heisenberg point, the spinons get dressed, but retain their fractionality. The entanglement spectrum of the HS state consists purely of the universal low spectral levels of the Heisenberg phase (all the other levels are shifted to infinity in $\xi$, corresponding to a largely degenerate $O$ eigenvalue level of $\rho_{A}$ ). From there, the entering logarithmic CFT corrections appearing as finite high-energy entanglement levels can be nicely observed upon interpolating between the ground states of the HS and the Heisenberg Hamiltonian. This underscores the fact that the HS and Heisenberg model belong to the same universality class, with the same relation between the Laughlin state and the Coulomb state in the FQHE.

We have introduced a suitable basis to fruitfully discuss entanglement spectra of quantum spin chains. Specifically, we have analyzed the $S=\frac{1}{2}$ Heisenberg ground state wavefunction and observe a universal set of states separated from higher levels by an entanglement gap persisting in the thermodynamic limit, bearing strong similarities to the Laughlin and Coulomb states in the FQHE. It is hence possible to determine the non-local structure of a spin system just by examining the ground state, without any reference to the concrete Hamiltonian, as we demonstrated for the Heisenberg state in its relation to the Haldane-Shastry state, as well the dimerization transition from the Heisenberg to the Majumdar-Ghosh state. From the present result and the FQH studies, a unified picture emerges: the low level structure of the entanglement spectrum reflects the properties of the lowenergy excitations of the system; it is in a one-to-one correspondence with the elementary excitations of the corresponding field theory. For FQH states, which are bulk gapped, an orbital cut as performed in [7, 9] is similar to a spatial cut due to the localized nature of the Landau orbitals on the sphere. The entanglement spectrum then reveals the nature of the low-energy excitations of the gapless edge system. For Heisenberg spin chains, the bulk is gapless, but the momentum cut does not longer correspond to a spatial cut, and the entanglement spec- 
trum reveals the nature of the bulk gapless excitations. In both cases, these low-energy excitations are described by $\mathrm{U}(1)$ bosonic field theory.

We thank N. Regnault for numerous discussions and for allowing us use of his cluster and for numerical help. We thank L. Balents for pointing out to us the vanishing leading logarithmic corrections at $\lambda_{c}$. We thank F.D.M. Haldane for numerous discussions and suggestions. RT was supported by a Feodor Lynen scholarship of the Humboldt foundation. DPA is grateful for the hospitality of the Princeton Center for Theoretical Science. BAB is supported by an Alfred P. Sloan Fellowship and by Princeton University start-up funds.

[1] R. Tao and F. D. M. Haldane, Phys. Rev. B 33, 3844 (1986).

[2] X. G. Wen, Phys. Rev. B 41, 12838 (1990).

[3] M. Hermele, T. Senthil, and M. P. A. Fisher, Phys. Rev. B 72, 104404 (2005).

[4] L. D. Faddeev and L. A. Takhtajan, J. Sov. Math. 24, 241 (1984).

[5] N. Andrei and J. H. Lowenstein, Phys. Rev. Lett. 43, 1698 (1979).

[6] P. Calabrese and J. Cardy, J. Stat. Mech. P06002 (2004).

[7] H. Li and F. D. M. Haldane, Phys. Rev. Lett. 101, 010504 (2008).

[8] F. D. M. Haldane, Phys. Rev. Lett. 51, 605 (1983).

[9] N. Regnault, B. A. Bernevig, and F. D. M. Haldane, Phys. Rev. Lett. 103, 016801 (2009).

[10] A. M. Turner, Y. Zhang, and A. Vishwanath, arXiv:0909.3119.

[11] L. Fidkowski, Phys. Rev. Lett. 104, 130502 (2010).

[12] R. B. Laughlin, Phys. Rev. Lett. 50, 1395 (1983).

[13] F. Pollmann and J. E. Moore, arXiv:0910.0051.

[14] M. Führinger, S. Rachel, R. Thomale, M. Greiter, and P. Schmitteckert, Ann. Phys. (Berlin) (2008).

[15] C. K. Majumdar and D. K. Ghosh, J. Math. Phys. 10, 1388 (1969).

[16] F. D. M. Haldane, Phys. Rev. B 25, 4925 (1982).

[17] B. A. Bernevig and N. Regnault, Phys. Rev. Lett. 103, 206801 (2009).

[18] F. D. M. Haldane, Phys. Rev. Lett. 60, 635 (1988).

[19] B. S. Shastry, Phys. Rev. Lett. 60, 639 (1988).

[20] B. A. Bernevig and F. D. M. Haldane, Phys. Rev. Lett. 100, 246802 (2008).

[21] The HS state, by virtue of being a homogeneous polynomial in the positions of the $\downarrow$ spins, always has $100 \%$ weight on the $M=K^{2}$ sector. Its component configurations in the crystal momentum basis also satisfy a 'squeezing' property [20], just like the Laughlin state (this is not obvious: the real-space HS wavefunction is written in terms of $K$ coordinates, while the Fourier transform sums over all $N=2 K$ positions that these coordinates can take on the unit circle). Away from the HS model, admixtures of components with $M=K^{2} \pm j N$ with $j=1,2, \ldots$, as well as 'un-squeezed' components with $M=K^{2}$, are generated. The entanglement spectrum is computed taking into account all other components but treating the full momentum as a good quantum number. 

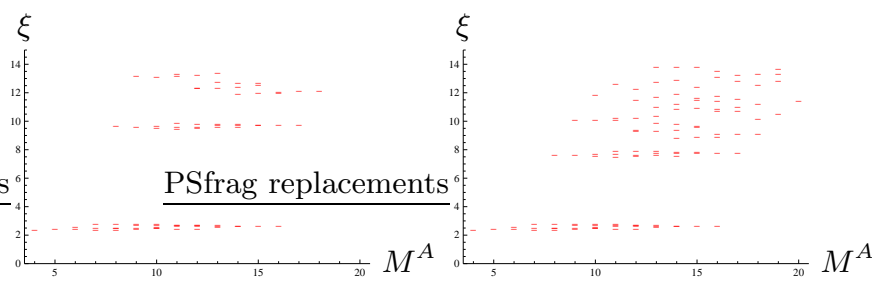

FIG. 4: Entanglement gap evolution of the interpolation Hamiltonian (5) with $N=16$ sites for the values $\gamma=0.75$ and 1.50. Note that high entanglement levels start to appear immediately away from the HS point, resulting in an entanglement gap. Already at $\gamma=1.5$, the spectrum already closely resembles the Heisenberg spectrum.
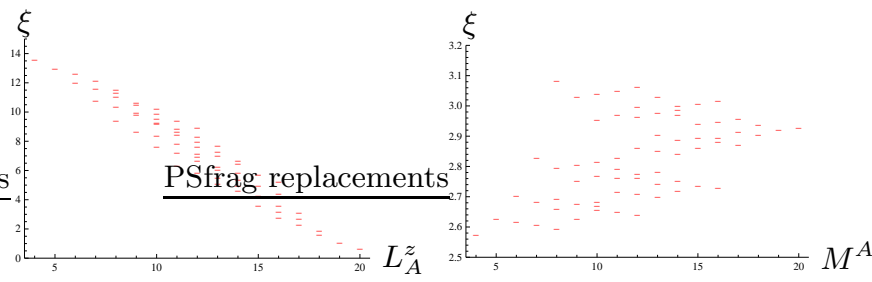

FIG. 5: Entanglement spectra of the $\nu=\frac{1}{2}$ Laughlin (left) and HS (right) states for systems with 9 particles (or downspins). The spectra consist solely of low level states; the entanglement gap is infinity. The edge mode counting exactly matches the prediction from the CFT. Both spectra possess exactly the same structure, while the HS spectrum - in absolute values of entanglement levels - is much flatter.

\section{Supplementary material:}

(i) Logarithmic corrections from the Haldane-Shastry point. We show how the entanglement spectrum changes from the Haldane-Shastry point to the Heisenberg point. This is best accomplished by the interpolation Hamiltonian

$$
H_{\mathrm{int}}=J_{0} \gamma^{2} \sum_{i<j} \frac{\vec{S}_{i} \cdot \vec{S}_{j}+\frac{1}{2}}{2 \sinh ^{2}\left(\gamma\left|z_{i}-z_{j}\right| / 2\right)},
$$

where the Haldane-Shastry limit is reached for $\gamma \rightarrow$ 0 . From the viewpoint of CFT, as discussed in the manuscript, the change in the entanglement spectrum relates to the logarithmic corrections entering from the conformally critical HS point. We find that the states enter in a flat band structured fashion corresponding to different orders of corrections, as shown in Fig 4.

(ii) Correspondence of Haldane-Shastry and Laughlin spectrum. As discussed in the manuscript, the HaldaneShastry state and the Laughlin state basically have the same entanglement spectrum, which only differs in terms of the manifold where the states are defined. Accordingly, the state counting exactly matches. However, the Laughlin levels spread over a much bigger range of entanglement energy, while the Haldane Shastry levels form an extremely flat band (Fig. 5).

(iii) Convergence of Lanczos algorithm. The biggest system size are computed by diagonalizing the spin Hamiltonian in a hardcore boson model in momentum space. The distribution of off-diagonal elements imposes a considerable challenge for Lanczos optimization convergence to generate the eigenstate. Exploiting all symmetries available in momentum space, the biggest sizes considered $(N=22)$ are matrices of dimension 3848879 with $\mathcal{O}\left(10^{9}\right)$ scattering elements. Convergence within a standard error of $\mathcal{O}\left(10^{-8}\right)$ for the Lanczos regeneration of the eigenstate demanded up to 50000 iterations.

(iv) Fitting procedures for the entanglement gap. There are slightly different ways to define the entanglement gap, all of which yield very similar results. The convention used in the paper is

$$
\Delta:=\operatorname{Min}_{m \in\left[M^{A}\right]}\left\{\operatorname{Min}_{\xi}\left\{\xi_{m}^{\text {generic }}\right\}-\operatorname{Max}_{\xi}\left\{\xi_{m}^{\text {universal }}\right\}\right\},
$$

i.e. the minimal gap between the highest low-energy universal level and lowest high-energy generic level of all different momentum sectors of $M^{A}$. Alternatively, one can define the entanglement gap globally over all different momentum sectors $\Delta_{1}:=\operatorname{Min}\left\{\xi^{\text {generic }}\right\}-\operatorname{Max}\left\{\xi^{\text {universal }}\right\}$. The latter can be further generalized to a gap $\Delta_{n}$ where instead a just taking the lowest generic level, we average over the lowest $n$ generic levels. If there were a spectral flow of only a single mode closes the gap, the difference in $\Delta_{1}$ and $\Delta_{n}$ would be able to detect it. For the cases considered in the manuscript, however, we only observe a collective rise or lowering of levels sets extending over all $M^{A}$ subsectors, which is why all these gap definition nearly conincide in their result.

(v) Spectral flow for the dimerization transition. A movie (movie-j1j2.gif) of the spectral flow from the Heisenberg point to the Majumdar Gosh point is added to the arxiv source files. 
This figure "movie-j1j2.gif" is available in "gif" format from: http://arxiv.org/ps/0912.0028v2 